$$
\text { "stankov" — 2007/2/15 — 17:23 — page 339 — \#1 }
$$

\title{
Some problems of solving linear equation with fractions
}

\author{
Gordana Stankov
}

\begin{abstract}
The aim of this paper is to offer some possible ways of solving linear equations, using manipulative tools, in which the "-" sign is found in front of an algebraic fraction which has a binomial as a numerator. It is used at $8^{\text {th }}$ grade.
\end{abstract}

Key words and phrases: early algebra teaching, learning difficulties, psychological aspects of concept formation, manipulative materials.

ZDM Subject Classification: C30, D70, E40, H20, H30, U60.

\section{Introduction}

Solving linear equations, particularly those with a "-" sign in front of the algebraic fraction and with a binomial as a numerator, is a challenge for secondary school students. The official Hungarian mathematics curriculum does not include this type of equations for $8^{\text {th }}$ grade students. However, taking into consideration that this kind of equation can occur in the secondary school entrance examinations, in addition to this they are traditionally given at this age at Hungarian schools I included them into my own teaching material for this grade for the academic year 2004/05. In this article, I shall report my initial positive teaching experiences in connection with this type of equation.

I am a teacher in the Serbian minority school in Budapest, Hungary, which teaches both primary and secondary school students. The official language in the school is Serbian, but during the lessons we use both languages: Serbian and Hungarian, because in the same class there are students who do not have

Copyright (C) 2006 by University of Debrecen
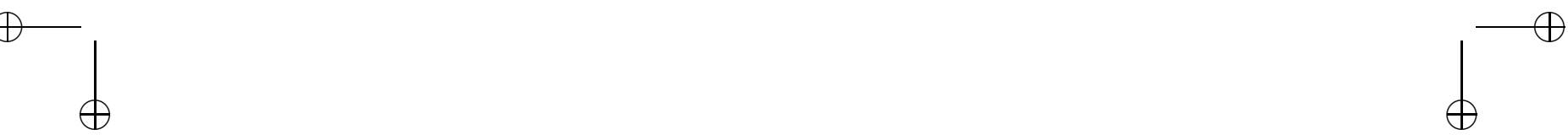
an appropriate command of the Serbian language. Some students attend our school with an aim to learn the Serbian language. Trying to help my students to overcome these language difficulties and enable them to adopt teaching material, I use a lot of gestures and visual aids in my teaching. While manipulating objects, the students are urged to use everyday Serbian language, which they have already learned, in order to explain mathematical relationships, operations and their own thoughts, as they are still not very familiar with technical expressions.

The average size of a class in our primary school (age 6-13) is about 10 students. This small number of students enables me to take an individual approach with every student, so I can deal with their individual problems.

In the spring of 2003, following the Hungarian official curriculum for the $6^{\text {th }}$ (3 girls, 5 boys) grade, I taught my students alternative methods of solving linear equations: trial and error through substitution of values for the unknown, as well as, cover up method. After that, the introduction into formal equation solving procedure, using real scales followed. The following year the formal equation solving was practiced. As solving the equations with the "-" sign in front of parentheses generally represents a problem for students, I decided to pay special attention to this problem and to introduce them to my students by gradually giving them tasks starting from easier to more difficult ones. While learning the rule of removing parentheses we used visual tools and referential meanings of algebraic expressions (Stankov, [8]), which can be either numerical or situational.

An algebraic expression can represent a relationship between numbers in general, which we refer to as numerical meaning. The numerical meaning for identity $a-(b+c)=a-b-c$ would be: When we subtract a number which is by " $c$ " bigger than number " $b$ " from number " $a$ " we get the same number as when we first subtract number " $b$ " from number " $a$ " and afterwards subtract number " $c$ " from the result obtained. The situational meaning of identity mentioned can be: Two girls, who finally had same number of apples, started from following situations: Mary had " $a$ " apples and she gave simultaneously " $b$ " apples to her sister and " $c$ " apples to her brother. Whereas, Ana initially had " $a$ " apples too, from which she ate " $b$ " apples and then gave " $c$ " apples to her mother.

Students solved equations with a "-" sign in front of parentheses without fractions (for example: $5-(x+3)=2 x-1$ ) and equations with algebraic fractions in which numerators were monomial (for example: $\frac{x}{2}+5=6+\frac{x}{3}$ ). 


$$
\text { "stankov" — 2007/2/15 — 17:23 — page } 341 \text { — \#3 }
$$

\section{Solving equations in the $8^{\text {th }}$ grade}

The starting point: evaluation of the level of knowledge

In the academic year 2004/05, we started dealing with linear equations particularly those with "-" sign in front of the algebraic fraction, the numerator of which is a binomial. All of the students ( 3 girls and 5 boys) had average mathematical skills. In order to establish the level of knowledge and their ability to use knowledge acquired earlier, in solving more complex equations, I set them the following 20 minute test:

(1) Which sign can replace the square in the equality: $5-(2 x-3)=5-2 x \square 3$ ?

Solve the following equations:

(2) $2 x-(x-3)=2 x+1$

(3) $8-\frac{x+4}{2}=3$

(4) $5 x-\frac{x-6}{2}=21$

Based on the material covered in the $6^{\text {th }}$ and $7^{\text {th }}$ grade I expected the following solutions:

(1) In the first task, the rule of removing parentheses when preceded by a "_" is used.

(2) In the second task the rule mentioned above is used: $2 x-x+3=2 x+1$. One of the variations of this can be either subtraction of $2 x$ to both sides of the equation or addition of $x-3$ to both sides (we observed that $x-3$ is a separate single whole).

(3) The third task was new for the students. Following the example of solving the equations containing an algebraic fraction, it is possible to multiply the equation by the denominator providing that they observe that the term $x+4$ represents one whole (temporarily). This binomial should be put into parentheses in the equation which does not contain a fraction any more.

Another solution would be to represent the fraction mentioned as a sum of two fractions. Having done that students should apply the rule of removing the parentheses preceded by a "-" sign. In this way, we should get a recognizable equation with algebraic fractions with monomials as numerators.

The third solution would be the "cover up" method meaning that they should determine the value of the fraction itself. 
(4) In the fourth task, it is possible to use the first and the second approach in order to solve the third task but it is impossible to use the "cover up" method.

Results of the test

(1) The first task was successfully solved by all of my students.

(2) For the second task only one student made a mistake. The solution he offered was as follows:

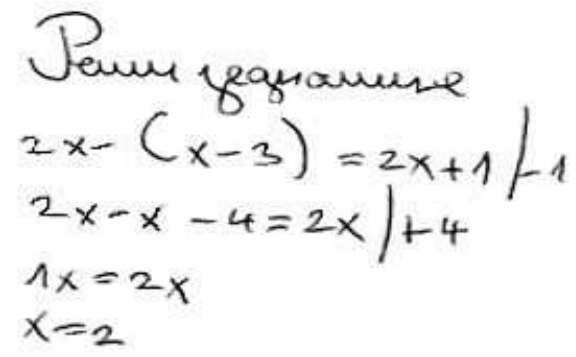

This solution indicates many problems. The student disregards the parentheses and approaches the task as if they were redundant. In the second step, the student does not add 4 to the right-hand side of the equation but correctly adds on the left side of the equation. Following this set of errors, the student gets $1 x=2 x$ and finally makes a wrong conclusion: $x=2$.

A girl used this approach:

$$
\begin{aligned}
& \text { 2) Reusu jorvalinie } \\
& 2 x-(x-3)=2 x+1 \\
& 2 x-(x-3)=2 x+1 /+(x-3) \\
& 2 x-(x-3)+(x-3)=2 x+1)+x-3 \\
& 2 x=3 x-2 \quad(-3 x \\
& x=-2(1-1) \\
& x=2
\end{aligned}
$$

The student seems to have no confidence in her knowledge. Although she was able to successfully solve the first task, she failed to apply the same rule of removal of parentheses in the remaining questions. She understands that $x-3$ is an object, as well as a process. She did not check her solution. 


$$
\text { "stankov" — 2007/2/15 — 17:23 — page 343 — \#5 }
$$

As my students had never dealt with problems of the type 3 and 4 , naturally, they made a lot of mistakes.

Dealing with the third exercise, a boy applied the method of substitution of values for the unknown and got the correct result.

One student managed the problem by using the "cover up" method. He omits to check as well. Here is his solution:

$$
\text { 3. } \begin{aligned}
& 8-\frac{x+4}{2}=3 \\
& 8-3=5 \\
& \frac{x+4}{2}=5 \\
& 10: 2=5 \\
& x+4=10 \\
& 6+4=10 \\
& x=6
\end{aligned}
$$

The rest of the class multiplied the equation by 2 . There was one single girl who considered the binomial as a whole and put it into parentheses but forgot to check the solution.

The rest of the class made the following typical mistake:

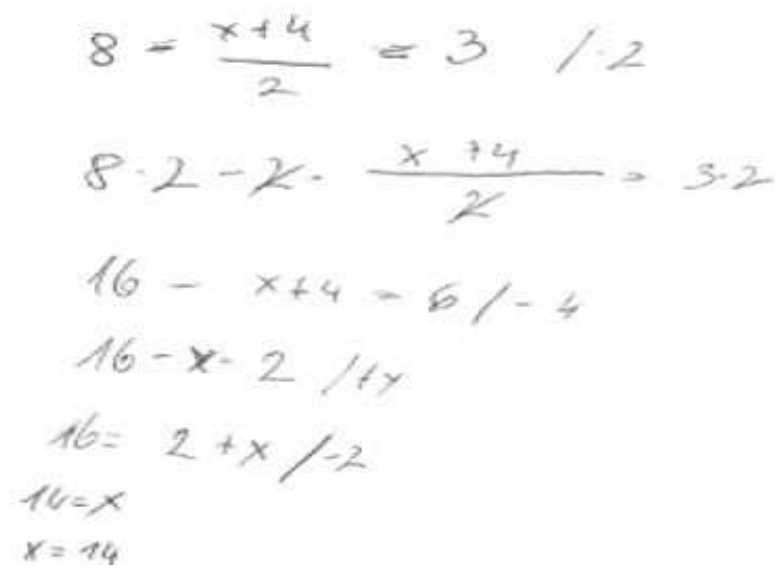




$$
\text { "stankov" — 2007/2/15 — 17:23 — page 344 — \#6 }
$$

Nobody checked the result in spite of the fact that it would show them the error.

The fourth equation was also a problem and the mistakes were analogical to the previously mentioned ones. The only correct solution was given by the girl who also found the solution of the third equation. Here is her solution:

$$
\begin{gathered}
5 x-\frac{x-6}{2}=21 / \cdot 2 \\
5 x-2-\frac{(x-6)}{x} \cdot z=21 \cdot 2 \\
10 x-x+6=42 /-6 \\
9 x=36 / 9 \\
x=4
\end{gathered}
$$

She considers the numerator to be a single whole and also correctly uses the rule of removing the parentheses. She has confidence in how to approach to the task by doing two steps simultaneously (calculates $10 x-x$ on the left side of the equation and adds -6 to the both sides).

\section{Supporting principles of the teaching method}

Skemp [7] differentiates between instrumental and relational understanding: the first one means that the student knows how to do things and the other one means that the student, as well as having this knowledge, also knows why the knowledge works. Very often students' understanding of algebra is instrumental. They manipulate algebraic symbols by using rules learned by heart. During my classes I try to support the development of students' relational understanding. By using my own teaching material I have an intention to develop the relational understanding of my students.

We should deal with fractions as well, because one student made a specific kind of mistake: $\frac{x-6}{2}=x-3$ and thus created a so-called malrule. Matz [5] says that students create malrules: In this situation the correct rule is: $\frac{x \cdot 6}{2}=x \cdot 3$. From the rule already mentioned, students derive the false general rule that any operational sign can replace the multiplication sign. Finally, the student actually applies a "-" sign instead of the "." sign and as a result she gets the following 


$$
\text { "stankov" — 2007/2/15 — 17:23 — page 345 — \#7 }
$$

malrule: $\frac{x-6}{2}=x-3$. My second intention is to fight against the creation of the malrules.

In the third task it was possible to determine the value of the fraction, whereas it was impossible in the fourth one. To proceed with the solution (in the forth task) we have to treat the fraction as a quotient by introducing new objects: $x-6$ (numerator) and 2 (denominator), or as a difference using new objects: $\frac{x}{2}$ (fraction with a variable as a numerator) and $\frac{6}{2}$ (fraction without variable). The third intention of this method is to shift the point of view as to recognize the algebraic expressions with their alternative meanings (Gray and Tall [4], Resnick [6], Stankov [8]).

\section{Elements of the teaching method}

\section{Cover up method}

When the task is: Solve the following equation $8-\frac{x+4}{2}=3$, the main difficulty for students connected to this method is that they cannot decide which part of the equation should be covered. I had to remind my students that using pieces of paper (of different shapes and colors that I gave them) and asking questions in such a case, is always helpful:

(1) What is the last operation before we get the number on the right side of an equation?

(2) How do we get the number on the right side of the equation?

They were able to vocalize the first appropriate question for our equation. They covered the expression $\frac{x+4}{2}$ by rectangular piece of paper and noted $8-\square=3$. The new equation was a simple one, so the students formulated the second question easily: What number should be subtracted from 8 in order to get 3 ? The answer was 5 and they made a note: $\square=5$. "What conclusion can be made?" I asked.

The answer was $\frac{x+4}{2}$ is equal to 5 . Consequently they got the new equation $\frac{x+4}{2}=5$ asking an analogical question, and covering $x+4$ by a circular paper they got first $\frac{\varrho}{2}=5$, and then $x+4=10$. Finally they concluded that $x=6$.

Checking: $8-\frac{10}{2}=3$.

It is impossible to solve the equations like the fourth one using the cover up method.

Expressing the fraction with binomial in numerator as the sum (difference) of 2 fractions: 
Although my students are able to add algebraic fractions with monomials as numerators easily, only one of them, solving the $4^{\text {th }}$ task, applied the reverse way of thinking and used the transformation: $\frac{x-6}{3}=\frac{x}{3}-\frac{6}{3}$. This transformation could be useful because the students would transform the $4^{\text {th }}$ equation into the equation: $5 x-\left(\frac{x}{2}-\frac{6}{2}\right)=21$ that they are already familiar with. I decided to present it to my students.

Giving referential meaning to the expression $\frac{x+4}{2}$

According to Resnick [6] algebraic expressions have referential meanings they can recall a relationship among quantities in some situation. My students are urged to use manipulative tools and referential meanings of the expression $\frac{x+4}{2}$. The issue is how the expression $\frac{x+4}{2}$ can be written down as a sum of 2 fractions.

After giving each of the students 6 fruit candies and 4 toffies I set them the task: There are 6 fruit candies and 4 toffies on the table, divide them justly, with your friend, so that both of you get the same number of candies of both kinds. Write down the appropriate transformation. The students did the division and mathematized the question by numerical expression $\frac{6+4}{2}=\frac{6}{2}+\frac{4}{2}$.

The task that followed was used to motivate conclusions without the actual manipulation of objects.

I raised the question: How can we justly divide in two parts a box of candies of one kind (the box contains $x$ number of candies), and an additional 4 candies of another kind. All the students were able to successfully solve the problem by saying that we should divide candies from the box into equal parts as well as remaining 4 candies, so they wrote: $\frac{x+4}{2}=\frac{x}{2}+\frac{4}{2}$.

In the similar way the student made the conclusion that $\frac{x-4}{2}=\frac{x}{2}-\frac{4}{2}$. (Once again we used fruit candies and four pieces of paper which represented the debt of one toffie. The question followed: divide the fruit candies and the debt with your friend justly).

\section{Using paper strips}

At the same time, we represented the expressions $\frac{x+4}{2}$ and $\frac{x}{2}+\frac{4}{2}$ using paper strips (the measurement unit was a square).

We started from the representations of $x$ and number 4 :

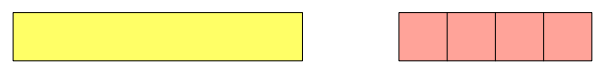




$$
\text { "stankov" — 2007/2/15 — 17:23 — page } 347 \text { — \#9 }
$$

We represented addition $x+4$ by sticking strips to one another. As a result of this process we got the $x+4$ object.
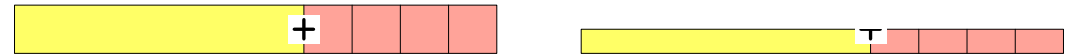

When we divided it by 2 (process) we got the result $\frac{x+4}{2}$ (object).

After this we represented $\frac{x}{2}+\frac{4}{2}$ (concept) by "adding" $\frac{4}{2}$ to the half of $x$. We started with $\frac{x}{2}$ and $\frac{4}{2}$.

By comparing the length of representations of and $\frac{x}{2}+\frac{4}{2}$ students realized that they were equal and they noted $\frac{x+4}{2}=\frac{x}{2}+\frac{4}{2}$. In the similar way using the strips the students came to the conclusion that $\frac{x-4}{2}=\frac{x}{2}-\frac{4}{2}$.

One student who used the malrule was urged to realize herself that the transformation $\frac{x+4}{2}=x+2$ (as well as the transformation $\frac{x-4}{2}=x-2$ ) was incorrect.

Giving her an opportunity to represent both of the expressions by paper strips and to compare them, she was able to realize herself that they were of different lengths.

During the exercise, the students practiced the use of alternative meanings of algebraic expressions.

\section{Formal equation solving}

In order to emphasize that the nominator of algebraic fraction represents a single whole, the students were urged to put the nominator into parentheses. Whereas, by using the paper strips it was indicated that the representation of the expression $10 x-(x-6)$ is not of the same length as the representation of the expression $10 x-x-6$ (see the second image on the page 342 ).

\section{Post-test}

During 3 lessons the students practiced exercises mentioned previously and solved equations such as the $3^{\text {rd }}$ and $4^{\text {th }}$ one. Students were encouraged to apply all the three previously mentioned methods when solving the equations. Among them cover up method was used whenever it was possible. After a week students had to solve the following equations as the post-test: 


$$
\text { "stankov" — 2007/2/15 — 17:23 — page 348 — \#10 }
$$

$$
\begin{aligned}
& 6-\frac{x+3}{6}=4 \\
& 4 x-\frac{x-6}{2}=7+3 x \\
& 3 x-\frac{2 x-5}{3}=3+2 x
\end{aligned}
$$

All the solutions were correct. When solving these equations two of the students got the correct solution in all the three equations mentioned by expressing algebraic fraction in the form of the sum of 2 fractions. Here is one of them:

$$
\begin{aligned}
& 4 x-\frac{x-6}{2}=7+3 x / \cdot 2 \\
& 4 x \cdot 2-\left(\frac{x}{2}-\frac{63}{x_{1}}\right) \cdot 2>14+6 x \\
& 8 x-x+6=14+6 x \\
& 7 x+6=14+6 x /-6 x \\
& x+6=14 /-6 \\
& x=8
\end{aligned}
$$

All the others used the formal balance method:

$$
\begin{aligned}
& \text { 3. } \\
& 3 x-\frac{2 x-5}{3}=3+2 x / \cdot 3 \\
& 8 x-2 x+5=9+6 x \\
& 7 x+5=8+6 x /-5 \\
& 7 x=4+6 x /-6 x \\
& x=4
\end{aligned}
$$

Unfortunately nobody checked the solutions, so I have to take care of this problem in the future. 


$$
\text { "stankov" — 2007/2/15 — 17:23 — page 349 — \#11 }
$$

\section{Delayed test}

The very same 20-minute test mentioned as the first one on the page 341 was repeated in December 2005.

Due to the summer holiday and the fact that the curriculum for the first term of the grammar school does not prescribe any kind of equation solving, these kind of exercise was not practiced for over half a year. After this period the first two tasks were successfully solved by all of the students, however the check of the second task was performed only in two cases.

Having completed the test I interviewed each of the students individually and asked them why the "+" sign substitutes the $\square$. Three of my students answered that they applied the rule of removing the parentheses in front of the "-" sign: $a-(b-c)=a-b+c$. One of the five similar explanations follows:

Teacher: What made you substitute $\square$ by "+" sign?

Student: I considered it.

Teacher: Describe your thoughts, please.

Student: We subtract the number which is smaller then $2 x$ from 5 . If we subtract $2 x$ from 5 , we subtracted more. To keep both sides equal we have to add 3 .

The same student that previously used the cover up method in order to give the correct solution for the third task, reached for the same method again. Four of the students represented algebraic fraction as a sum of 2 fractions. One of the solutions was incorrect as the student made an error when adding at the end of the task. Three of the students used the formal method of solving equations. Two of them applied the method as in the third example on the page 344 (one of them was a girl who gave the good solution at the initial test). Finally, one student made the same mistake as the one in the second example on the page 343. Four of the students did not check the solution; among them were those whose results were wrong.

The forth task was correctly solved by 5 students who used the method of representing algebraic fraction as a sum of 2 fractions. Two of them checked the solution. The formal method of solving equations was used by those students who also applied this method in the task 3 . The same student repeated the same mistake from the third task and he did not check the solution. Three of the students checked the solution of the forth task. 


$$
\text { "stankov" — 2007/2/15 — 17:23 — page 350 — \#12 }
$$

\section{Conclusions}

Despite the good test results it seems that this type of equation, when dealing with algebraic fractions with binomial as a numerator and a "-_ sign in front of the fraction, is difficult and only appropriate in simple cases for this age group. Based on the results of the post-test and the delayed test I dare to make the conclusion that my students adopted the method of representing algebraic fraction as a sum of 2 fractions. I can also state that they rather prefer this method than using the formal method of solving equations. The paper shows that two of the students used the method of representing algebraic fraction as a sum of two fraction in the post-test, while four students used it in the third and five students in the forth task when doing the delayed test. In my opinion the experience that obtained manipulating the candies and paper strips considerably contributed to the more frequent and comfortable use of this method. The use of the manipulative tools urged the students to realize how the algebraic fraction can be represented as a sum of 2 fractions. After a certain period of time it was easier for students to recall this reasoning compared to the formal method of solving equations.

Using the referential meanings of algebraic expressions is a helpful tool for reconstruction and building up the rules of algebraic transformations as well as for self-correction of algebraic transformations.

This kind of individual work with students can be (in a class of 30 or more students have to be) supported by some computer programs (see for example Aspetsberger \& Fuchs [1], Berta [2], Dominik \& Fuchs [3], Varga-Neofotistos [9], Vásárhelyi [10]).

\section{References}

[1] K. Aspetsberger, K. Fuchs, Computer Algebra Systeme für den Mathematikunterricht. Praxis der EDV/Informatik, Verlag Jugend \& Volk, 1996.

[2] T. Berta, Combination of traditional and computer based tools in mathematics education, ZDM 2003, Vol. 35, no. 1, 2003.

[3] A. Dominik, K. Fuchs, MATHEMATICA - Palettes - A Methodical Way to Provoke Students into Using Mathematical Strategies, in: ICTMT 4, CD-ROM, 1999.

[4] M. Gray, D. Tall, Duality, Ambiguity and Flexibility: A Proceptual View of Simple Arithmetic, The Journal for Research in Mathematics Education 26, no. 2, 1994, $115-141$. 


$$
\text { "stankov" — 2007/2/15 — 17:23 — page 351 — \#13 }
$$

[5] M. Matz, Towards a process model for high school algebra errors, in: Intelligent tutoring system, (D. Sleeman, J. S. Brown, eds.), Academic Press, New York, 1982, $25-50$.

[6] L. B. Resnick, E. Cauzinille-Marmeche, J. Mathieu, Understanding algebra, in: Cognitive processes in mathematics, (J. A. Sloboda, D. Rogers, eds.), Clarendon Press, Oxford, 1987, 169-203.

[7] R. Skemp, Relational understanding and instrumental understanding, Arithmetic Teacher 26, no. 3, 1978, 9-15.

[8] G. Stankov, The use of concrete and visual representation in teaching early algebra in order to avoid basic difficulties, in: Positionenen - Mathematikdidaktik in Entwicklung, (K. J. Parisot, É. Vásárhelyi, eds.), Abacus Verlag, Salzburg, 2005, 188-203.

[9] I. Varga-Neofotistos, Diagnostik von Rechenfehlern am Beispiel der Division - ein Computerprogramm, Dissertation, University of Salzburg, 2002.

[10] É. Vásárhelyi, Combination of traditional and computer based tools as a strategy for problem solving, in: Creativity and Mathematics Education, Münster, 1999, $163-166$.

GORDANA STANKOV

PH.D. STUDENT

UNIVERSITY OF DEBRECEN

HUNGARY

E-mail: sgordonka@yahoo.com

(Received February, 2006) 